\title{
Acetonitrile Adsorption on Polycrystalline Platinum: An In situ Investigation Using Sum Frequency Spectroscopy
}

\author{
S. Beau Waldrup and Christopher T. Williams* \\ Department of Chemical Engineering \\ University of South Carolina, Columbia, SC 29208
}

\section{Supporting Information}

\section{Gas Phase Adsorption of Acetonitrile}

Gas phase adsorption of acetonitrile on Pt was studied and is compared with that of neat liquid acetonitrile adsorption. This was accomplished by following the procedure as outlined in the text. The resulting spectrum (Figure S1) shows the primary $v_{\mathrm{C}=\mathrm{N}}$ vibration located at 2233 $\mathrm{cm}^{-1}$. This is only slightly downshifted from the peak position observed in the neat liquid (2238 $\mathrm{cm}^{-1}$ ) indicating similar adsorption in both phases.

\section{Exploring Solvation Effects}

The solvation of acetonitrile in ethanol, methanol and water was explored using ATR-IR. The spectra were taken using a Nicolet Nexus 670 instrument, with a frequency resolution of 2 $\mathrm{cm}^{-1}$. The experiments were conducted by first flowing neat acetonitrile over a Germanium (Ge) waveguide and taking a spectrum of bulk acetonitrile using the dry Ge waveguide as the background. The concentration of the flowing liquid was then altered by adding small amounts of the solvent and allowing the system to come to equilibrium, usually less than 5 minutes. Spectra were taken at intervals from 100 to 10 volume $\%$ in $10 \%$ increments. The peak position of the $v_{\mathrm{C} \equiv \mathrm{N}}$ stretch of acetonitrile was recorded and plotted as a function of acetonitrile concentration in Figure S3. The data clearly show a shift in the peak position of the $v_{\mathrm{C} \equiv \mathrm{N}}$ stretch of acetonitrile with increasing concentration of each of the solvents used in this study. 


\section{Figure Captions}

Figure S1 Comparison of the gas phase adsorbed acetonitrile versus the liquid phase neat acetonitrile. The liquid phase data is given by circles and the gas phase data by squares. The models are shown as lines.

Figure S2 Plot of the peak position of the $v_{\mathrm{C} \equiv \mathrm{N}}$ stretch of acetonitrile versus concentration. The data for ethanol is represented by squares, methanol by triangles and water by circles. The lines are to help guide the eye. 


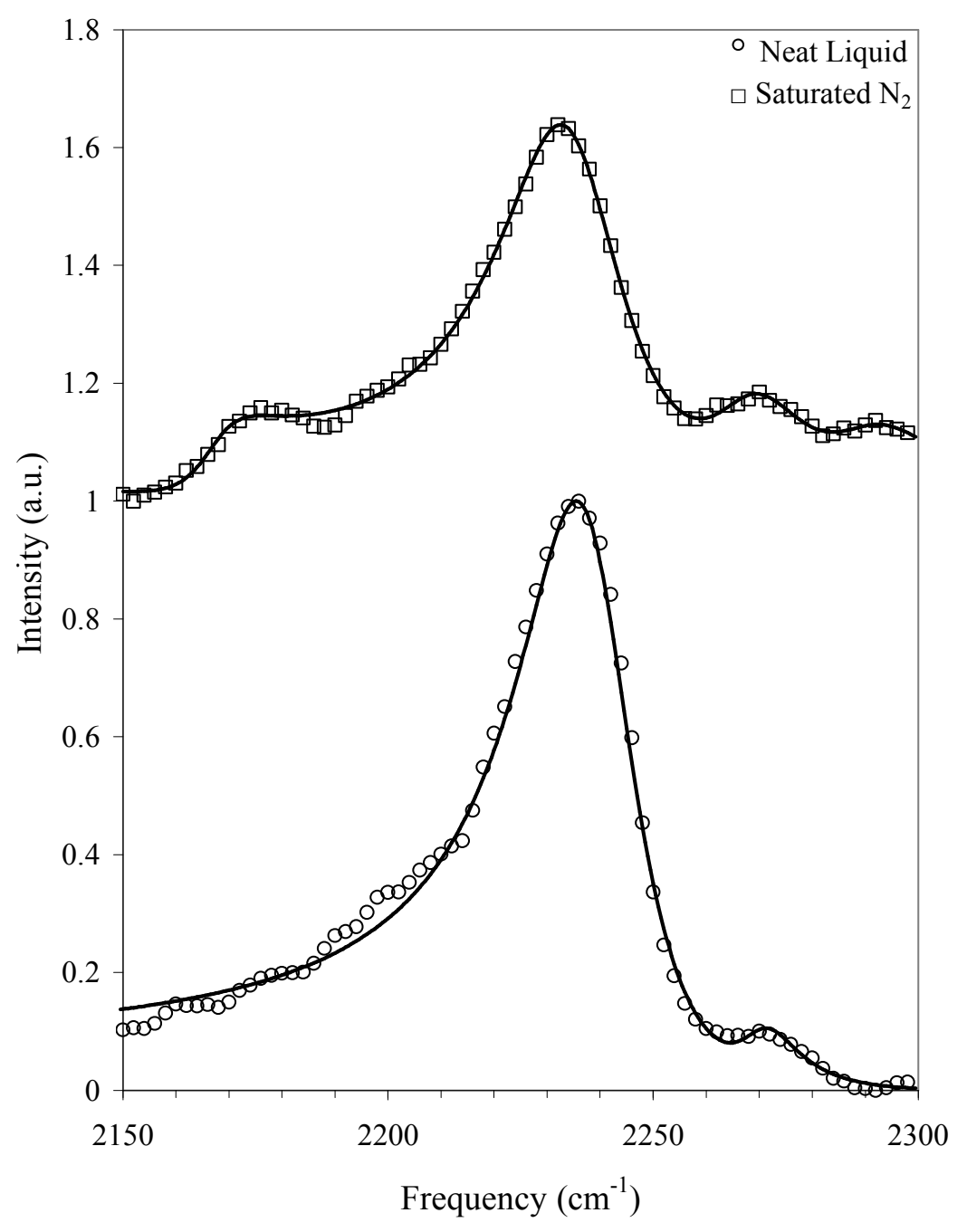

Figure S1 


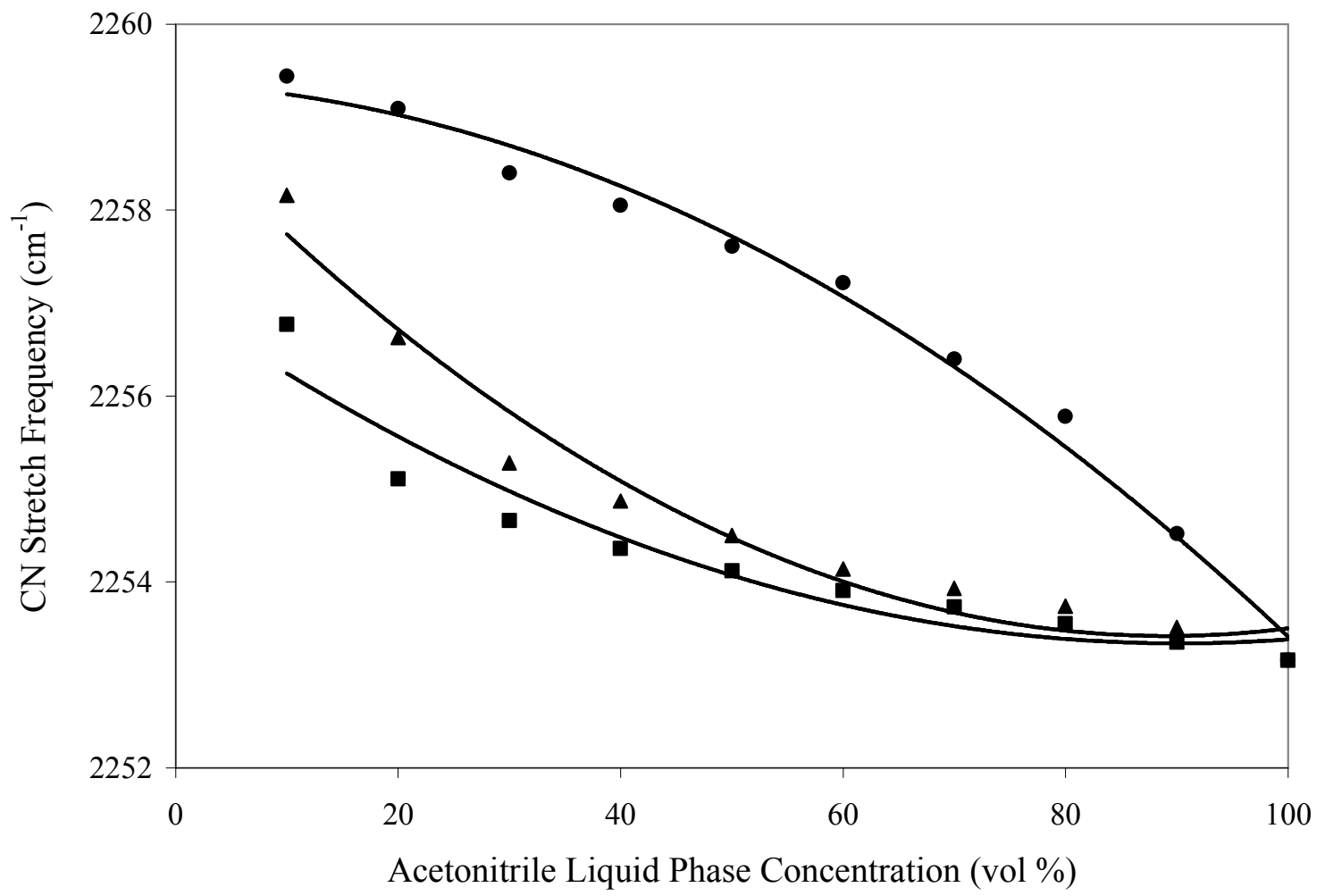

Figure S2 